\title{
Translational Chinese Medicine: A Way for Development of Traditional Chinese Medicine
}

\author{
Xuegang Sun ${ }^{1}$, Donglan Lin ${ }^{1}$, Weikang $\mathrm{Wu}^{2^{*}}$, Zhiping $\mathrm{Lv}^{{ }^{*}}$ \\ ${ }^{1}$ The Key Laboratory of Molecular Biology, State Administration of Traditional Chinese Medicine, \\ School of Traditional Chinese Medicine, Southern Medical University, Guangzhou, China \\ ${ }^{2}$ The Institute of Integrated Traditional Chinese and Western Medicine, Sun Yat-sen University, Guangzhou, China

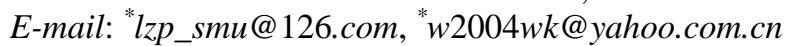 \\ Received October 22, 2011; revised November 23, 2011; accepted December 3, 2011
}

\begin{abstract}
Translational Chinese medicine is one of the latest developing fields in traditional Chinese medicine. In this paper, we discuss the " $3 \mathrm{w}$ " namely, "what is", "why to advance", "how to carry out" and the significance of translational Chinese medicine. To overcome the innate drawbacks of traditional Chinese medicine (TCM), the basic theory of TCM had better be refreshed. The safety and efficacy of classic formulae and therapy experience of TCM should be evaluated based on strict quality control and reaffirmed with evidence based medicine. The significance of translational Chinese medicine is to transform Chinese medicine into a balanced, personalized medicine with sound safety, good efficacy and strict quality control.
\end{abstract}

Keywords: Translational Medicine, Traditional Chinese Medicine, Quality Control, Efficacy, Safety

\section{Introduction}

TCM, which is the quintessence of the Chinese cultural heritage, has made an everlasting contribution to the survival, propagation and prosperity of all ethnic groups in China, thereby enhancing the fertility and prosperity of the nation. In 2008, the Ministry of Science and Technology jointed together by other fifteen Ministries, includeing Ministry of Health, State Administration of Traditional Chinese Medicine, and State Food and Drug Administration, etc, collectively issued the "Outline of Traditional Chinese Medicine Innovation and Development Plan (2006-2020)". The outline explicitly pointed out that inheritance, innovation, modernization, and internationalization of TCM would be the four basic tasks for a considerable period of time. Thereby, combination and integration of classic heritage of TCM with innovations of life science would be the necessary avenue to develop TCM. The essence of the avenue is to translate the ancient knowledge of Chinese medicine into clinical effectiveness. Therefore, we propose a new research model translational Chinese medicine, to utilize global scientific and technological resources, to provide evidence and to facilitate Chinese medicine globalization.

\section{What Is Translational Chinese Medicine?}

“Translational medicine” is a trendy term being increase- ingly used to describe the wishing of biomedical researchers to ultimately help patients [1]. It is the link between "bench and bedside" and a scientific approach that bridges laboratory experiments through clinical trials, to actual health-care applications.

"Translational medicine" (or "translational research") describes a uni-directional effort to test in humans novel therapeutic strategies developed through experimentation. "Translational Chinese medicine" is a two-way street: one way is going from the bedside to the laboratory with observations made in clinical practices of TCM and another way is driving back to bedside to cure and collect Data of evidence-based medicine. The former might be more constructive, focusing scientific thinking and providing more practical information [1]. The evidence from the latter is more objective. So, the first step is to fill up the gap between the empiricism of Chinese medicine and molecular models of life science. The second step is to bridge up the safety, effectiveness of Chinese medicine to bedside and community.

\section{Why Translational Chinese Medicine?}

The safety, efficacy, quality, availability, preservation, and further development of TCM are often questioned, because of its seemingly under developed scientific knowledge of TCM. So, some translational work need be done 
to improve the scientific contents of TCM.

\subsection{The Transcendental Basic Theory Needs Translational Renovation}

Influenced by the idea of natural philosophy and cognitive theory of "analogy based classification" and "deductive reasoning", the basic theory of TCM has natural philosophy neuclear under a transcendental robe. The five zang-organs theory is an example in that it includes characters of intangibility, formality and ideality. For example, brain is the most important organ, however, it is included among the "the oddly Fu organs". Pancreas and testicles are not even listed in zang-organ system at all. The tongue is supplemented to eyes, ears, mouth, and nose to be the five orifices. The long summer is fabricated to cater the five seasons. The five elements theory is the promodial model to promulgate the "natural law" for zang-organs. The formation of zangfu-organs theory is a process that changes the big notes of general proposition into small coins [2]. This kind of exchange could be finished overnight and the result theory could neither be verified nor be it falsifiable. Therefore, although the theory of five elements occupies a lot of space in the textbook, nearly no progression was made over thousands of years [3]. So, some new knowledge, such as crosstalks between signal transduction network, had better to fuse into holism idea of TCM and make it progress with the advance of live science.

\subsection{The Safety and Efficacy of TCM Prescription Need Translational Evaluation}

In 2000, World health organization (WHO) formulated the "General Guidelines for Methodologies on Research and Evaluation of Traditional Medicine" and also warned that "with the tremendous increase in the use of traditional medicines worldwide, the safety, efficacy, and quality control of herbal medicines and traditional procedure-based therapies has become an important concern for both healthcare authorities and the public". Ten years later, traditional herbal medicinal products directive (THMPD) declared that the sale of all Chinese herbal medicines has been banned within the European Union (EU) from 1st April 2011 as a result that not a single Chinese herbal medicine has been approved and licensed in the EU. The aim of THMPD is to ensure the safety and efficacy of herbal medicines and prescriptions in EU. The underlying word is that although the efficacy of TCM has been "field-tested" by tens of thousands of people for hundreds of years, it does not necessarily mean that it is really safe and it indeed works. So, what we need today is to find solid data to repeat what the TCM prescription claimed to be safe and effective for therapeutic practice. It is only on this basis that further scientific studies can be done. For example, meta-analyses showed that there was insufficient evidence of good quality in proving the efficacy of traditional Chinese patent medicines used for treating ischemic stroke [4]. Moreover, this is a common phenomenon in most TCM research. Therefore, high quality TCM researches with adequate information and solid data on safety and efficacy evaluation are crucial. Translational Chinese medicine is the way to bring about high level evidence-based resources.

\subsection{The Empiricism in Clinical Practices Needs Translational Validation}

Theory of TCM is a pragmatic tool to explain the results of clinic practices [5]. It is also the carrier of clinical experiences sedimented generation after generation. For example, syndrome differentiation is the landmark of Chinese medicine clinical practice. However, as the diagnosis tools in TCM are less tangible, the TCM practitioner depends more on experience, technique, intuition and insight. Thus, the gradual development of Chinese medicine is based on constant accumulation and summary of experience in clinical practice [6]. Therefore, syndrome differentiation has always been lying in expirism even after thousands of years of evolution. The practitioner can only make a fuzzy, holistic, and black box evaluation on the syndrome. So, more work should be done to document the experience with the language of life science and could be recognized and permitted by the international society.

\section{How to Carry out Translational Chinese Medicine}

\subsection{Refreshing the Basic Theory of TCM}

The natural philosophy confers basic theory of TCM with clear-cut humanities features and philosophical meanings that put a strangle hold on its progression with the development of modern life science. For example, the fiveelement theory is no longer adequate to explain the functionality and relationship of five zang-organs. However, if the five-element theory were specifically depleted from TCM, some TCM experts would say that it is no longer TCM anymore. So, the rational crux of basic theory of TCM should be reserved. It had better to be refreshed by absorbing the essence from the fast development of contemporary medical science but not reconstruct it to be Chinese medicine without tradition. For instance, it is a well-documented fact that the dysfunction of hypothalamic-pituitary-adrenal (HPA) axis and 
locus coeruleus-norepinephine (LC-NE) system plays an important role in chronic stress-induced depression [7]. In TCM, the corresponding dipiction is that the failure of liver-organ to dominate conveyance and dispersion results in liver-stagnancy syndrome. We can therefore establish the relationship between liver and brain. The hyperactive liver-Qi might be related with the dysfunction of certain nucleus. It is therefore understandable that liver-soothing and Qi-dispersing herbs or formulae could be prescribed to cure depression.

\subsection{Reassure Safety and Efficacy of TCM Prescrition with Strict Quality Control}

In the United States, a Phase I study of PHY906 in combination with capecitabine was conducted in patients with advanced pancreatic cancer [8] and a similar Phase I/II study of PHY906 in combination with capecitabine in advanced hepatocellular carcinoma (HCC) were conducted [9]. Phytomics quality control is a characteristic methodology adopted, which combines chemical analysis, bioresponse analysis, and animal pharmacology to determine batch-to-batch reproducibility [9]. Formula is the major method and final carrier for TCM pricription. The safety of formula is a case involving human life and thus should to be treated with the utmost care. So, we propose a new concept "formulomics", a strategy similar to phytomics with strict quality control and established clinical efficacy. It is a unified platform integrating: 1) standard extraction process with fixed material basis; 2) exact treatment effects with information-rich bioresponse fingerprints; 3) clear target based on absorbed bioactive compound (ABC) [10] and regulatory mechanism based on statistical pattern comparisons; and 4) compatibility mechanism of major ingredients based on immunoaffinity Chromatography $[11,12]$. The research model is different from phytomics that it includes all classic TCM formulae; not only phyto-derived formulae and it would study the compatibility mechanism. Those classic formulae, especially formulae from "Treatise on Febrile Diseases" and "Synopsis of Golden Chamber", are of choice for formulomics research. It is an open platform and a translational medicine strategy to translate the classic formulae of TCM into new clinical drugs. It focuses on quality control and efficacy evaluation for it's the most important factors for the standardization of TCM [13].

\subsection{Reaffirm the Efficacy of TCM with EBM}

Although the history of traditional Chinese medicine can give people more confidence in terms of "evidence based practice", their efficacy are still be queried by the international society. To make the efficacy of TCM meet the modern criterion, a commonly accepted standard of TC$\mathrm{M}$ syndrome had better to be established in the pattern of combining diseases with syndrome differentiation of their syndromes. At the same time, the good practices of TCM clinical guidelines and clinical pathways and a grading system for TCM evidence had been developing [14]. As the Chinese EBM Centre has taken the responsibility to promote the modernization of TCM in 2007, it is time to combine these standards with EBM to reaffirm the efficacy of TCM. Furthermore, widely accepted animal models that could mimic the pathogenesis of diseases with syndrome differentiation for evaluation efficacy of TCM are a must. Objective evaluation criterion and reverse verification by classic Chinese formula are also needed. For example, chronic unpredictable mild stress model is a widely accepted model for depression and also for liver -Qi stagnation. Forced swimming test, sucrose preference test, and open field test would be performed, and xiaoyaosan could be chosen as the reverse verification medicine. The unified system would reduce the confusion and chaos in TCM efficacy research.

\section{Significance of Translational Chinese Medicine}

\subsection{To Translate Chinese Medicine to Balanced Medicine}

The persue of translational Chinese medicine is to be balanced medicine: balance between maximizing safety, effectiveness, long term value, and minimizing the risk of side-effects. Translational clinical practice system (TCPS) [15] is of choice to strike the balance. The aim of TCPS is to facilitate the practitioners' skills in translating the multifaceted inter-relationship of scientific evidence, patient preferences and values, practitioner experience and judgment, clinically relevant outcomes, and ethical practice parameters into substantial healthcare improvements for their patients [16]. To accomplish TCPS in T$\mathrm{CM}$, The first stage is to ensure the safety of herbal medicines with strich quality control. Then EBM with the pattern of combining diseases with differentiations of their syndromes is the second stage to ensure its efficacy and patient preference.

\subsection{To Translate Chinese medicine to Personalized Medicine}

Chinese medicine is patient-centered in all ages. Syndrome differentiation is the promodial form of personalized medicine. Translational medicine is synonymous with "molecular medicine" or "personalized medicine" as all of them refer to the process of applying molecular insight 
into the clinical care of the patients [17]. Provided that large-scale data sets from genomics, proteomics, population genetics, and imaging drives research at an unprecedented pace, translational Chinese medicine is to integrate these data with the safety and efficacy of TCM to take deeper insights into syndrome differentiation and formulae [18]. The gene studies pertaining to pharmacogenetical variations in response to Chinese medicine response, optimum dose for adequate response, pathophysiology changes of a particular formula, patient population predisposition to adverse effects would provide the pharmacogenetic basis of drug effect variations in patients. In the long run, the application of pharmacogenetics in Chinese medicine will significantly contribute to the individualization of drug therapy, known as personalized Chinese medicine.

As the science matures, the empiricism of medical practice is replaced by mechanism-based targeted diagnostics and therapeutics. Molecular sciences, including the evolving science of biomarkers, have catalyzed the development of diagnostic and therapeutic platforms tailored to the individual patient profiles, paving the way for the deployment of personalized Chinese medicine. Therefore, it is time to advance the concept of translational Chinese medicine to make Chinese medicine a personalized and balanced medicine, with sound safety, good efficacy and strict quality control.

\section{Acknowledgements}

This Project was supported by grants from the National Natural Sciences Foundation of China (No. 81173168, 30973699).

\section{References}

[1] S. P. Mankoff, C. Brander, S. Ferrone and F. M. Marincola, "Lost in Translation: Obstacles to Translational Medicine,” Journal of Translational Medicine, Vol. 2, No. 1, 2004, pp. 14-18.

[2] X. G. Sun, Q. Liu, Y. Y. Zhao and Y. Cai, “Confirmation, Falsification, or Hermeneutics?” Journal of Nanjing University of Traditional Chinese Medicine (Social Science Edition), Vol. 8, No. 1, 2007, pp. 30-33.

[3] J. Xu and Y. Yang, "Traditional Chinese Medicine in the Chinese Health Care System,” Health Policy, Vol. 90, No. 2-3, 2009, pp. 133-139. dx.doi.org/10.1016/j.healthpol.2008.09.003

[4] B. Wu, M. Liu, H. Liu, W. Li, S. Tan, S. Zhang, et al., "Meta-Analysis of Traditional Chinese Patent Medicine for Ischemic Stroke,” Stroke, Vol. 38, No. 6, 2007, pp. 1973-1979.

[5] X. G. Sun, "The Negative Influences of Pragmatism on Traditional Chinese Medicine (TCM)," Medicine and
Philosophy, Vol. 19, No. 9, 1998, pp. 19-21.

[6] B. Liu, Y. Zhang, J. Hu, L. He and X. Zhou, “Thinking and Practice of Accelerating Transformation of Traditional Chinese Medicine from Experience Medicine to Evidence-Based Medicine,” Frontier Medical, Vol. 5, No. 2, 2011, pp. 163-170. dx.doi.org/10.1007/s11684-011-0143-9

[7] R. L. Hauger, V. Risbrough, R. H. Oakley, J. A. Olivares-Reyes and F. M. Dautzenberg, "Role of CRF Receptor Signaling in Stress Vulnerability, Anxiety, and Depression," Annals of the New York Academy of Sciences, Vol. 1179, No. 2009, pp. 120-143.

[8] M. W. Saif, F. Lansigan, S. Ruta, L. Lamb, M. Mezes, K. Elligers, et al., "Phase I Study of the Botanical Formulation PHY906 with Capecitabine in Advanced Pancreatic and Other Gastrointestinal Malignancies," Phytomedicine, Vol. 17, No. 3-4, 2010, pp. 161-169.

[9] Y. Yen, S. So, M. Rose, M. W. Saif, E. Chu, S. H. Liu, et al., "Phase I/II Study of PHY906/Capecitabine in Advanced Hepatocellular Carcinoma,” Anticancer Research, Vol. 29, No. 10, 2009, pp. 4083-4092.

[10] X. Huang, F. Qin, H. M. Zhang, H. B. Xiao, L. X. Wang, X. Y. Zhang, et al., "Cardioprotection by Guanxin II in Rats with Acute Myocardial Infarction Is Related to Its Three Compounds,” Journal of Ethnopharmacology, Vol. 121, No. 2, 2009, pp. 268-273. dx.doi.org/10.1016/j.jep.2008.10.029

[11] R. Tilton, A. A. Paiva, J. Q. Guan, R. Marathe, Z. Jiang, W. van Eyndhoven, et al., "A Comprehensive Platform for Quality Control of Botanical Drugs (Phytomicsqc): A Case Study of Huangqin Tang (HQT) and PHY906," Chinese Medicine, Vol. 5, 2010, pp. 1-15. dx.doi.org/10.1186/1749-8546-5-30

[12] Y. Sun, Y. Dong, H. J. Jiang, T. T. Cai, L. Chen, X. Zhou, et al., "Dissection of the Role of Paeoniflorin in the Traditional Chinese Medicinal Formula Si-Ni-San against Contact Dermatitis in Mice," Life Sciences, Vol. 84, No. 11-12, 2009, pp. 337-344.

dx.doi.org/10.1016/j.lfs.2008.12.023

[13] Y. Wang, X. Huang, F. Qin, P. Ren, Z. Zhu, R. Fan, et al., "A Strategy for Detecting Optimal Ratio of Cardioprotection-Dependent Three Compounds as Quality Control of Guan-Xin-Er-Hao Formula,” Journal of Ethnopharmacology, Vol. 133, No. 2, 2011, pp. 735-742. dx.doi.org/10.1016/j.jep.2010.11.006

[14] Y. Li, T. Wu, H. Shang and K. Yang, "Strategies for Promoting the Development of Evidence-Based Medicine in China,” Journal of Evidence-Based Medicine, Vol. 2, No. 1, 2009, pp. 47-52. dx.doi.org/10.1111/j.1756-5391.2009.01012.x

[15] M. U. Naidu, "Promise of Translational Medicine: An Evidence-Based Therapeutics," Indian Journal of Pharmacology, Vol. 43, No. 2, 2011, pp. 103-104. dx.doi.org/10.4103/0253-7613.77332

[16] G. K. Merijohn and M. G. Newman, “The Translational Clinical Practice System: A Way to Implement the Evidence-Based Approach in the Dental Office,” Journal of 
the California Dental Association, Vol. 34, No. 7, 2006, pp. 529-539.

[17] A. Terzic and S. A. Waldman, "Translational Medicine: Path to Personalized and Public Health," Biomarkers in Medicine, Vol. 4, No. 6, 2010, pp. 787-790. dx.doi.org/10.2217/bmm.10.101

[18] M. Wehling, “Translational Medicine: Science or Wishful Thinking?” Journal of Translational Medicine, Vol. 6, No. 3, 2008, pp. 1-3. 